\title{
On grain growth kinetics in two-phase polycrystalline materials through Monte Carlo simulation
}

\author{
K R PHANEESH* ${ }^{*}$ ANIRUDH BHAT ${ }^{\dagger}$, GAUTAM MUKHERJEE ${ }^{\ddagger}$ and K T KASHYAP ${ }^{\dagger}$ \\ Department of Mechanical Engineering, MS Ramaiah Institute of Technology, Bangalore 560 054, India \\ ${ }^{\dagger}$ Department of Mechanical Engineering, PES Institute of Technology, Bangalore 560 085, India \\ $\ddagger$ Manish Apartments, Convent Road, Off Residency Road, Bangalore 560 001, India
}

MS received 3 July 2011; revised 15 February 2013

\begin{abstract}
Monte Carlo Potts model simulation was carried out on a 2D square lattice for various surface fractions of second phase particles for over $\mathbf{5 0 , 0 0 0}$ iterations. The observations are in good agreement with known theoretical and experimental results with respect to both growth kinetics as well as grain size distribution. Further, the average grain size and the largest grain size were computed for various surface fractions which have indicated normal grain growth and microstructure homogeneity. The surface fraction of the second phase particles interacting with the grain boundaries $(\Phi)$, hitherto not computed through the simulation route, is shown to vary inversely as the average grain size due to Zener pinning.
\end{abstract}

Keywords. Monte Carlo method; Potts Q-states; metropolis algorithm; grain growth exponent; grain size distribution; Zener pinning.

\section{Introduction}

Generally, the final step in the thermo-mechanical processing of metallic materials is annealing which restores physical and mechanical properties of cold worked metals. Two important processes occur during annealing: primary recrystallization, driven by stored energy and grain growth, driven by the grain boundary surface energy. Since recrystallization and grain growth are the key factors involved in the control of grain size and primarily determine properties like strength, fatigue and formability, they have been subject of extensive investigations, both experimentally and theoretically, over many decades. There is a very interesting relationship between the conceptual basis of the Monte Carlo method as a simulation technique and the physical characteristics of grain growth. As far as both rely very much on statistics and randomness, the method seems to represent the phenomenon very well (Landau and Binder 2005). Therefore, with recent advances in computing, the scene has shifted to mathematical modelling and simulation studies where powerful computers have led the way in the study of microstructure evolution.

Grain growth is a process through which the average grain size in polycrystalline materials increases with time, primarily driven by the excess surface free energy associated with the grain boundary. This energy drives the grain boundaries to migrate towards their centre of curvature, thereby reducing its local surface area and resulting in some kind of a competition where some grains shrink and disappear while some

\footnotetext{
*Author for correspondence (krphaneesh@gmail.com)
}

others grow to fill the volume previously occupied by the shrinking grains. Apart from the grain boundary surface energy, the other force which drives grain growth is-the difference in the volume free energy between the grains on either side of the grain boundary, which in turn is attributable to differences in chemical composition, crystal structure, elastic strain-energy density or crystal defect density (Frost and Thompson 1986).

When grain growth is driven by the reduction of grain boundary energy alone and in the absence of any impediments to curvature-driven grain boundary motion, it is expected that normal grain growth occurs.

Normal grain growth can be distinguished by two characteristics: (i) microstructural homogeneity where the largest grain size in a given microstructure is only $2.5-3$ times larger than the mean grain size and (ii) time invariance of the grain size distribution when plotted as a function of the normalized grain radius, $R / R_{\text {mean }}$, where $R$ is the radius of each grain and $R_{\text {mean }}$ the mean radius of all grains. Burke and Turnbull (1952) has given us the classic equation on grain growth kinetics where he proposes that the average grain size occurring during normal grain growth grows as:

$$
R_{\text {mean }}=k t^{n} \text {, }
$$

where $R_{\text {mean }}$ is the average grain diameter, $t$ the time and $k$ a constant that varies exponentially with temperature. The maximum value of $n$, known as grain growth exponent, is $0 \cdot 50$. However, this is rarely observed experimentally with most metals having $n$ well below 0.50 due to reasons such as grain boundary mobility, varying metal compositions, temperature and the limiting effects on grain growth due to 
impurities and solute atmospheres (Humphreys and Hatherly 2005).

In the present work, a thorough investigation is carried out to ascertain the value of grain growth exponent in monophase materials as well as two-phase materials containing various surface fractions of second phase particles. The grain size distribution and microstructure homogeneity would also be evaluated through simulation studies and the viability of such a study would be demonstrated.

\section{Monte Carlo method of simulation}

The Monte Carlo method is a probablistic computer simulation technique which is extensively used in statistical physics, but also used to study grain growth and related phenomena. While analytical models predict ensemble characteristics of microstructural evolution, computer simulations have helped to generate snapshots of the evolving microstructure with time (Holm and Battaile 2001). Using the computational version of metallography, both local and ensemble properties of the microstructure may be determined from these snapshots. Among a few computer simulation methodologies which have been evolved over the years, the Monte Carlo method is one of the prominent techniques employed to simulate microstructural evolution in crystalline materials.

The origin of the Monte Carlo simulation can be traced back to early 19th century during the development of models for studying ferromagnetic materials in the domain of solid state physics. The Ising (1925) model developed represented a magnetized material as a collection of spins where only two states were possible, i.e. up and down. This model was later improvised by Potts (1952) who allowed for $Q$ states, rather than only two, to accommodate each particle in the system. This method was further developed by von Neumann (1952) and Metropolis et al (1953) to study the diffusion of neutrons in fissionable materials. But adaptation of Monte Carlo technique using the Metropolis algorithm (Ising and Potts models) for the simulation of microstructure was first introduced by Anderson et al (1984) and Srolovitz et al (1984) for two-dimensional grain growth and extended to three-dimensional grain growth by Anderson et al (1989).

\subsection{Metropolis algorithm for Q-states Potts model simulation}

The procedure for Monte Carlo simulation of grain growth by using Metropolis algorithm is as follows: (i) a square matrix of size ' $N$ ', representing the cube lattice, is generated with $N^{2}$ elements or lattice sites. All lattice sites represent random numbers ranging from 1 to $Q$, where $Q$ indicates local crystallographic orientation, (ii) randomly throw $f$ percent of second phase particles into the matrix and inert its state value by considering a value for $Q$ other than $1: Q$, (iii) among the $N^{2}$ elements present in the matrix, a random site is chosen and is compared with all its nearest neighbouring elements which is eight in case of square lattice.

If $i$ is the element randomly picked and $j$ any neighbouring element that $i$ is compared with then,

$$
\begin{array}{ll}
\partial_{i j}=0 & \text { if } \quad i \neq j, \\
\partial_{i j}=1 & \text { if } \quad i=j,
\end{array}
$$

where $\partial_{i j}$ is the Kroneckar delta, a relative interaction energy value between one element and any other neighbouring elements. The Hamiltonian, which is defined as the sum of kinetic and the potential energy that a material possesses at any instant, is then calculated for the chosen element by the following relation

$$
\text { Hamilton }\left(E_{1}\right)=-J \sum_{1}^{n}[\partial(i, j)-1],
$$

where $\partial(i, j)$ are Kroneckar delta values for interaction between $i$ th and $j$ th matrix elements, $n$ the number of nearest neighbouring elements (eight, in our case) and $J(>0)$ is an interfacial energy constant of the system and (iv) the grain orientation corresponding to the chosen element is changed into a new random element in its place and the Hamiltonian $\left(E_{2}\right)$ is calculated again for the new element using the relation as in (2), giving the energy change, $\Delta E=E_{2}-E_{1}$.

Now if $\Delta E \leq 0$, the change is accepted, else if $\Delta E>0$, the change is rejected.

The entire steps from 1 to 4 form one iteration and is repeated ' $N$ ', $(N=$ matrix size $)$ number of times which constitutes one Monte Carlo step (MCS).

\section{Experimental-(hardware and software)}

All the experiments were carried out on a specially built system with 16 GB RAM, INTEL CORE i5-2500K-6M3.3GHZ processor and a Asus P8H67-MLE Motherboard B3 Model. A highly efficient, versatile and superfast code has been written on Java platform while close attention was paid to memory management so as to save time since simulations ran into a cumulative of more than a million Monte Carlo steps. For example, it took $\sim 2$ min to run 1000 Monte Carlo steps on a $1000 \times 1000$ matrix which involved $10^{9}$ iterations of the Metropolis algorithm.

\section{Results and discussion}

All simulations took place on a two-dimensional square lattice of matrix size $(N)$ of $1000 \times 1000$ with the number of $Q$ states being 64 using regular Monte Carlo algorithm rather than the modified Monte Carlo proposed by Radhakrishnan and Zacharia (1995), with the intention of keeping the simulation truly random. The second phase particles were all given a uniform size of one pixel and made static by giving a $Q$ value of zero.

Figure 1 shows variation of the average grain radius $\left(R_{\text {mean }}\right)$ with increasing Monte Carlo steps up to 50,000 


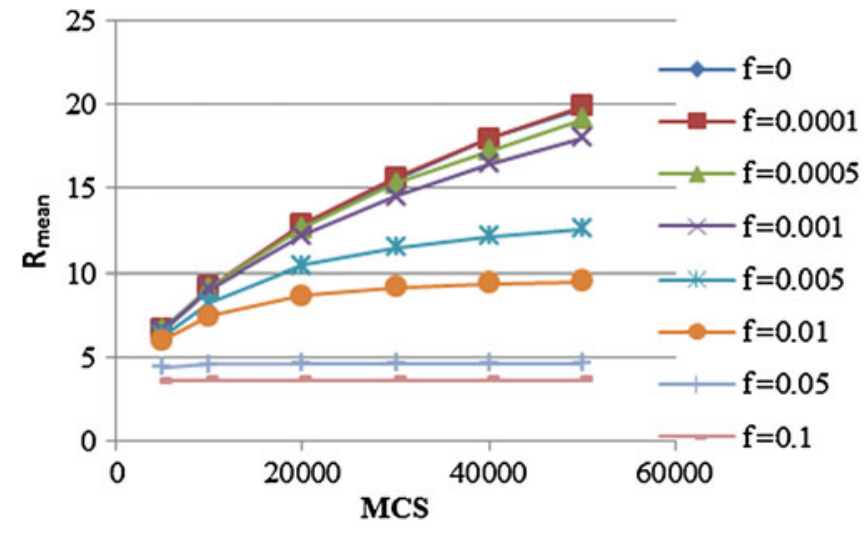

Figure 1. $R_{\text {mean }}$ (in pixels) vs MCS for $N=1000$ at $Q=64$.



Figure 2. Largest grain size (in pixels) vs MCS for $N=1000$ at $Q=64$.

cycles for various surface fractions of second phase particles $(f)$ including none. Individual grain areas were first computed and from the mean area $(A)$ equated to a circle, $R_{\text {mean }}$ was calculated as $\operatorname{sqrt}(A / \pi)$, with all dimensions in terms of pixels. It is very clear that the rate at which $R_{\text {mean }}$ increases with the iterations decreasing with the surface fractions with almost negligible growth taking place at higher values of $f$. At higher values of $f$, a larger percent of second phase particles interact with grain boundaries. Therefore, Zener pinning is more operative in such cases than at lower values and, hence, the fall in the rate of increase of the grain radius. Figure 2 shows variation of the largest grain size (LGS) with MCS for various values of $f$ and it mimics the relationship between $R_{\text {mean }}$ and MCS, which is typical of normal grain growth. Comparing figures 1 and 2, we also notice that LGS is only around 3 times the $R_{\text {mean }}$, for the respective surface fraction, which is also a characteristic of normal grain growth.

The grain growth kinetics can be analysed from figures 3 and 4 which show the variation of $R_{\text {mean }}$ vs MCS on a $\log$ scale and the variation of grain growth exponent $(n)$ vs $f$, respectively. Grain growth exponent values, obtained as slopes of $\log R_{\text {mean }}$ vs $\log$ MCS curves for a given $f$, represent the rate at which grains grow with increase in simulation

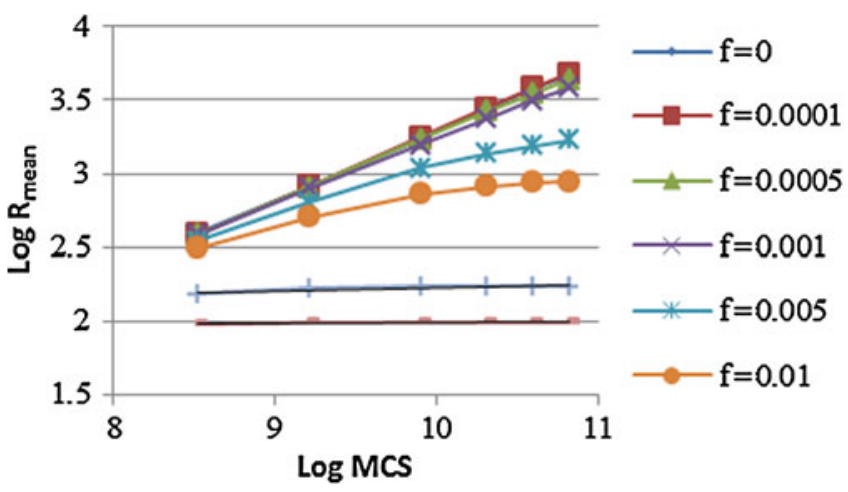

Figure 3. $\log R_{\text {mean }}$ vs $\log \operatorname{MCS}$ for $N=1000$ and $Q=64$.



Figure 4. Grain growth exponent $(n)$ vs surface fraction, $f$, for $N=1000, Q=64$.

time. Figure 4 shows that at $f=0$, the grain growth exponent shows a value of $n=0.48(m \approx 2)$ which is closest to the theoretically evaluated value of $n=0 \cdot 5$ (Louat 1974). But with increasing additions of second phase particles, the value of $n$ drops continuously to near zero, which is due to massive grain boundary pinning. From figure 3, it is evident that grain growth hardly takes place given the high surface fractions of particles deployed.

The percent fraction of the second phase particles lying on the grain boundaries $(\Phi)$, against MCS, was plotted for various surface fractions as shown in figure 5. $\Phi$ was calculated considering the inert particles which lay on the edges of each grain. It was observed that number of particles interacting with the grain boundary was high at higher surface fractions, while it came down with increasing MCS at lower surface fractions and stabilized towards higher Monte Carlo steps. We know from Gibbs-Thomson equation $(\Delta p=2 \gamma / R$, where $\Delta p$ is the pressure difference across a grain boundary, $\gamma$ the grain boundary energy and $R$ the grain curvature) and also from Gibb's free energy equation $\left(\delta_{\mathrm{g}}=V_{\mathrm{m}} 2 \gamma / R\right.$, where $\delta_{\mathrm{g}}$ is the free energy change, $V_{\mathrm{m}}$ the molar volume and the terms $\gamma$ and $R$ as in the previous equation) (Martin et al 1997) that the grain radius is inversely proportional to both the pressure difference across the grain boundary as well as Gibb's free energy change. This means that at lower values of $R$, both $\Delta p$ as well as $\delta_{\mathrm{g}}$ are higher and, therefore, the driving force for grain growth is greater. Since the grain 


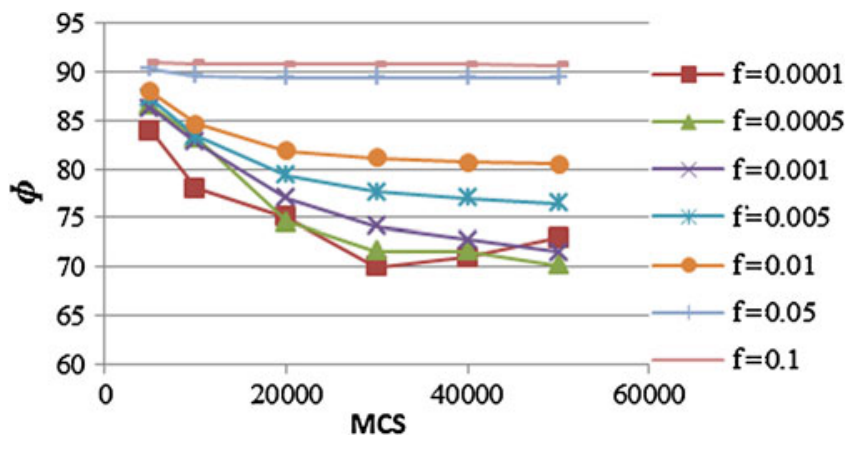

Figure 5. $\Phi$ vs MCS for $N=1000$ and $Q=64$.



Figure 6. Variation of $R_{\text {mean }}$, LGS and $\Phi$ vs $f$.

sizes and hence the grain radii are smaller at the beginning of grain growth, there is a relatively higher driving force which results in the second phase particles freeing themselves from the grain boundaries moving at higher velocities. As the iterations are increased, the driving force reduces while there is an onset of limiting grain size and $\Phi$ stabilizes due to both reasons. Figure 6 clearly shows how $R_{\text {mean }}$ and LGS drops off with the increase in surface fraction, while $\Phi$ increases with increasing $f$ implying inverse relationship with $R_{\text {mean }}$ and LGS.

A few snapshots of the simulated microstructures in figure $7(\mathrm{a}-\mathrm{c})$ (at MCS $=50,000$ and at various values of $f$ ) demonstrate the closeness to real ones. The micrographs show an equiaxed distribution of grain structure, showing static Zener particles in figure 7(b-c). Faceting which often results in square lattice simulation has been overcome to a good extent by considering a matrix of large size. It can also be observed that the grain sizes reduce on an average with increasing surface fraction of second phase particles at the given MCS of 50,000 steps and can be attributed to Zener pinning of the grain boundaries by the second phase particles. Self-similarity of evolving microstructures, which is a characteristic of grain growth, can also be observed in the three digital micrographs.
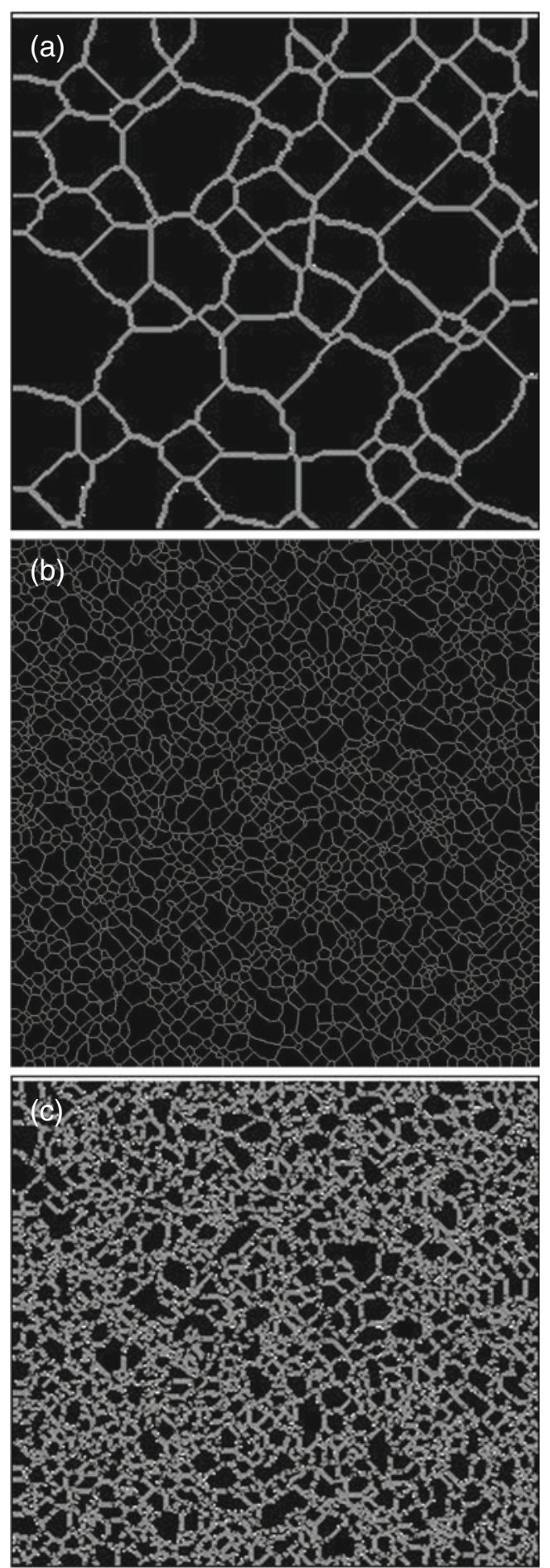

Figure 7. (a) $N=1000, Q=64, f=0$, (b) $N=1000, Q=$ 64, $f=0.005$ and (c) $N=1000, Q=64, f=0.05$.

\section{Conclusions}

(I) It is observed that the Metropolis algorithm forms excellent basis while Java provides excellent software support for studying microstructure evolution in polycrystalline materials. 
(II) Grain growth exponent was found to be $n=0.48$ in single phase material simulation, while the value dropped to near zero for a high surface fraction of second phase particles of $0 \cdot 1$.

(III) Grain size distribution, microstructure representation and microstructure homogeneity were very well achieved through simulation.

(IV) Percent fraction of second phase particles interacting with the grain boundaries, which is difficult to be evaluated in experimentation, was found to be much more than what was assumed by Zener in his original work.

\section{Acknowledgements}

We are indebted to the Visvesvaraya Technological University, Karnataka, which funded this project (No. VTU/Aca/2009-10/A-9/11417). We are also grateful to the following: Dr K Rajanikanth, Dr M K Muralidhara and Dr R Chandrashekar, all of MSRIT, Bangalore; Dr K N Balasubramanya Murthy, Principal, PESIT, Bangalore; and Dr C S Ramesh, Praveen and Anirudh, all are research scholars at the Centre for Nano-Composites Research, PESIT.

\section{References}

Anderson M P, Grest G S and Srolovitz D J 1989 Philos. Mag. B59 293
Anderson M P, Srolovitz D J, Grest G S and Sahni P S 1984 Acta Metall. 32783

Burke J E and Turnbull D 1952 Prog. Metal Phys. 3220

Frost H J and Thompson C V 1986 Computer simulation of microstructure evolution (ed) D J Srolovitz (Warrendale, PA: TMS-AIME) 15086 p. 33

Holm E A and Battaile C C 2001 JOM 5320

Humphreys F J and Hatherly M 2005 Recrystallization and related annealing phenomena (UK: Pergamon Press) 2nd ed, p. 335

Ising E 1925 Z. Phys. 31253

Landau P D and Binder K 2005 A guide to Monte Carlo simulations in statistical physics (UK: Cambridge University Press) 2nd ed, p. 1

Louat N P 1974 Acta Metall. 22721

Martin J W, Doherty R D and Cantor B 1997 Stability of microstructure in metallic systems, Cambridge solid state science series (Cambridge: Cambridge University Press) 2nd ed, p. 247

Metropolis N, Rosenbluth A W, Rosenbluth M N, Teller A H and Teller E 1953 J. Chem. Phys. 211087

Potts R B 1952 Proc. Cambridge Philos. Soc. 48102

Radhakrishnan B and Zacharia T 1995 Metall. Mater. Trans. A26 167

Srolovitz D J, Anderson M P, Sahni P S and Grest G S 1984 Acta Metall. 32793

Von Neumann J 1952 Grain shapes and other metallurgical applications of topology by C S Smith in Metal interfaces (Cleveland, Ohio: ASM) p. 108 